\title{
Low-cost Appliance Switching Circuit for Discarding Technical Issues of Microcontroller Controlled Smart Home
}

\author{
Mehedi Hasan 1, 2, *, Maruf Hossain Anik², Sumon Chowdhury², Sabbir Alam Chowdhury ${ }^{2}$, \\ Toufiqul Islam Bilash ${ }^{3}$, Sharnali Islam ${ }^{4}$ \\ ${ }^{1}$ IC Mask Design Department, Ulkasemi Pvt. Limited, Dhaka, Bangladesh \\ ${ }^{2}$ Department of Electrical and Computer Engineering, North South University, Dhaka, Bangladesh \\ ${ }^{3}$ Department of Electrical and Computer Engineering, Independent University, Dhaka, Bangladesh \\ ${ }^{4}$ Department of Electrical and Electronic Engineering, Dhaka University, Dhaka, Bangladesh
}

Email address:

mehedi.hasan01@northsouth.edu (M. Hasan),maruf.anik01@northsouth.edu (M. H. Anik),

sumon.chowdhury@northsouth.edu (S.Chowdhury), sabbir.alam@northsouth.edu (S. A. Chowdhury), mdbilash14@gmail.com (T. I. Bilash), sharnali.eee@du.ac.bd (S. Islam)

${ }^{*}$ Corresponding author

\section{To cite this article:}

Mehedi Hasan, Maruf Hossain Anik, Sumon Chowdhury, Sabbir Alam Chowdhury, Toufiqul Islam Bilash, Sharnali Islam. Low-cost Appliance Switching Circuit for Discarding Technical Issues of Microcontroller Controlled Smart Home. International Journal of Sensors and Sensor Networks. Vol. 7, No. 2, 2019, pp. 16-22. doi: 10.11648/j.jijsn.20190702.11

Received: July 27, 2019; Accepted: August 16, 2019; Published: August 26, 2019

\begin{abstract}
As technological advancement in recent years is bringing ease to our everyday life, smart home systems based on wireless networks and sensors are rapidly gaining importance. Research on microcontroller controller smart home systems has become popular due to its low cost and reliability. However, appliance switching capacity of microcontroller is quite low which limits the widespread use of such sort of systems. This paper introduces an external appliance switching circuit for microcontroller controller automated home which has the ability to control 208 appliances. This large appliance switching capability has been obtained by demultiplexing digital signals obtained from microcontroller board using a complex array of 4 to 16 line decoders. The proposed appliance switching circuit can be implemented with $60-70 \%$ less wiring cost in real time compared to the typical wiring technique in microcontroller controlled smart homes. Also, hardware implementation expenditure in this research has been reduced significantly compared to the existing designs. Moreover, the issue associated with existing microcontroller appliance switching scheme in case of incorporating manual switch has been solved. The proposed circuit is incorporated with a smart phone application by which the user sends commands to the microcontroller board using Bluetooth as the medium of wireless transfer. The proposed microcontroller controlled smart home system with external appliance switching circuit is quite cheaper than the existing systems which allows consumers to afford smart living with limited budget. Due to reduction of implementation cost and solution of major technical design issues incorporated with the existing microcontroller-based system been solved in this research work, microcontroller based smart home systems is expected to become a potential product in the market in near future.
\end{abstract}

Keywords: Smart Home System, Microcontroller, Arduino UNO, Smart Phone Application, Bluetooth

\section{Introduction}

Smart Home Systems or simply Smart Home means automation of our electrical and electronic devices of our house. Smart Home has achieved a great interest in the recent years and has become a popular topic of interest for researchers. By implementing Smart Home System in our home, numerous household activities of appliances can be controlled [1]. Depending on user's demand, various types of Smart Home Systems have been developed [2].

Early versions of Smart Home Systems used Dual Tone Multi Frequency (DTMF) technology [3]. However, DTMF technology-based appliance control system in home is not quite reliable since the system often result in false appliance switching. Recently, due to technological advancement in 
wireless sensors and smart devices, Smart Home System is heading towards a new dimension. Using smart phones to control home appliances is the most recent trend in Smart Home Systems [4]. Moreover, hand gesture controlled systems [5, 6] and voice controlled smart home system [7] are getting popularity nowadays. However, gesture controlled and voice controlled smart hoe systems require huge installation cost which makes these systems less affordable for people having low budget. Moreover, accuracy is a major problem in such sort of systems. Global System for Mobile (GSM) and Internet have made operating range of home automation systems global [8-10]. Internet based systems nowadays offers a wide range of smart functions. But GSM and Internet based smart home systems are sometimes unreliable due to speed issues. Moreover, operating cost of GSM and Internet based smart home systems are high due to SMS and Internet bills. Bluetooth based smart homes offer users smart living within low budget but they have limited range of operation [11]. Energy management in smart home have also become popular to optimize energy consumption which reduces electricity bills significantly [12].

Microcontroller controlled smart home systems have recently gained much interest among researchers [13]. Various smart home methodologies such as Gesture Controlled, Bluetooth based, GSM technology based, voice recognitionbased use microcontrollers for appliance switching $[5,11,14$, 15]. Microcontroller offers a low-cost implementation of smart home appliance control system. But the number of appliances that can be controlled by a microcontroller is very limited. Remote switching of appliances is the most basic and major function of a smart home. Due to having limited number of pins, microcontrollers don't have the ability of controlling a large number of appliances. Due to this reason, widespread use of microcontroller controlled cheap smart homes is halted. Hence, it is necessary to enhance the appliance switching capacity of microcontroller controlled smart homes.

This paper introduces a microcontroller controlled smart home system with an external circuit which has the capacity of controlling 208 appliances. The external circuit only needs 4 pins of microcontroller to handle this large number of appliances. The system incorporates a smart app with user-friendly Graphical User Interface (GUI) by which user can send commands to the microcontroller board. Bluetooth was used as the wireless medium from smart phone to microcontroller board.

\section{Literature Review of Existing Microcontroller Controlled Appliance Switching Circuits}

Microcontroller controlled Smart Home Systems are gaining much importance nowadays due to its simplicity and low-cost implementation. Various smart features and complicated functions such as voice-controlled lighting, flame and gas monitoring, automatic ventilation etc. can be implemented by microcontroller controlled Smart Home Systems [16].

Typical microcontroller-controlled appliance switching systems consist of a smart phone app, a wireless device or system such as Bluetooth, Wi-fi, Internet etc. and relays to switch on/off appliances [11, 17]. Block diagram of conventional microcontroller controlled smart home for appliance switching is presented in figure 1. Microcontroller based appliance switching other than using smart app such as Gesture Controlled, GSM controlled, voice controlled etc. utilizes the same technique for appliance switching by connecting microcontroller pins directly to relay $[5,14,15]$. The major problem for such sort of systems is appliance switching capacity since microcontroller has limited number of pins. For instance, ATmega328 microcontroller-controlled system presented [16] can control only 12 appliances. ATmega2560 microcontroller-based system presented in the study [18] can control 52 appliances which provides a better option.

Another major problem of microcontroller-based appliance control is inability of switching appliance manually. These systems cannot incorporate manual switches by which appliances can be switched on/off manually. Hence, it is necessary to overcome this problem.

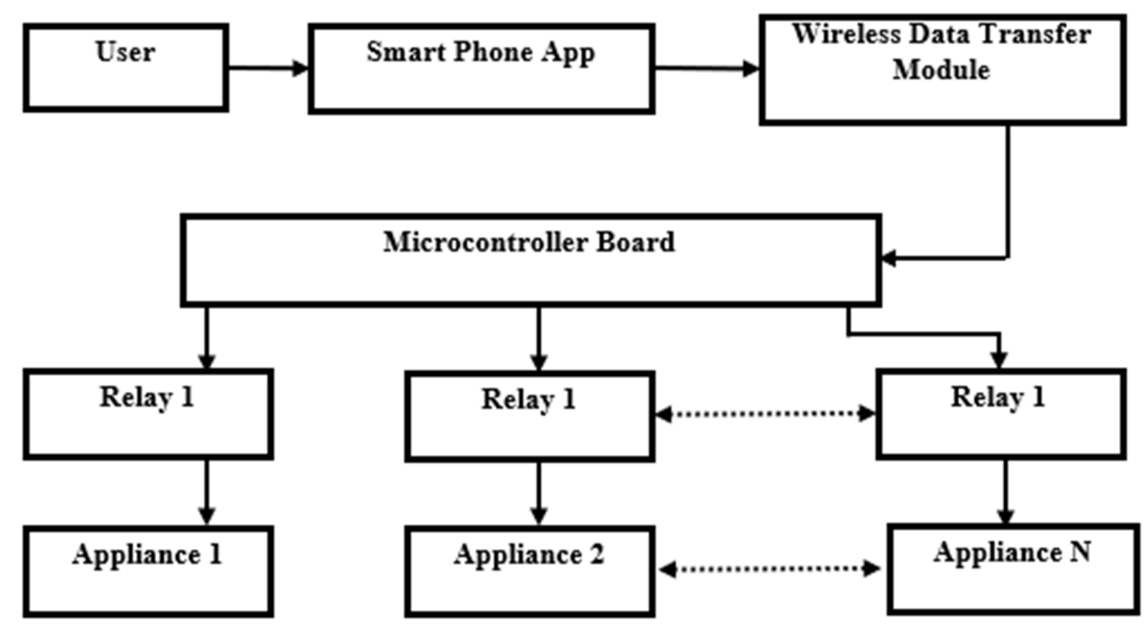

Figure 1. Block diagram of conventional microcontroller-controlled appliance switching circuit. 

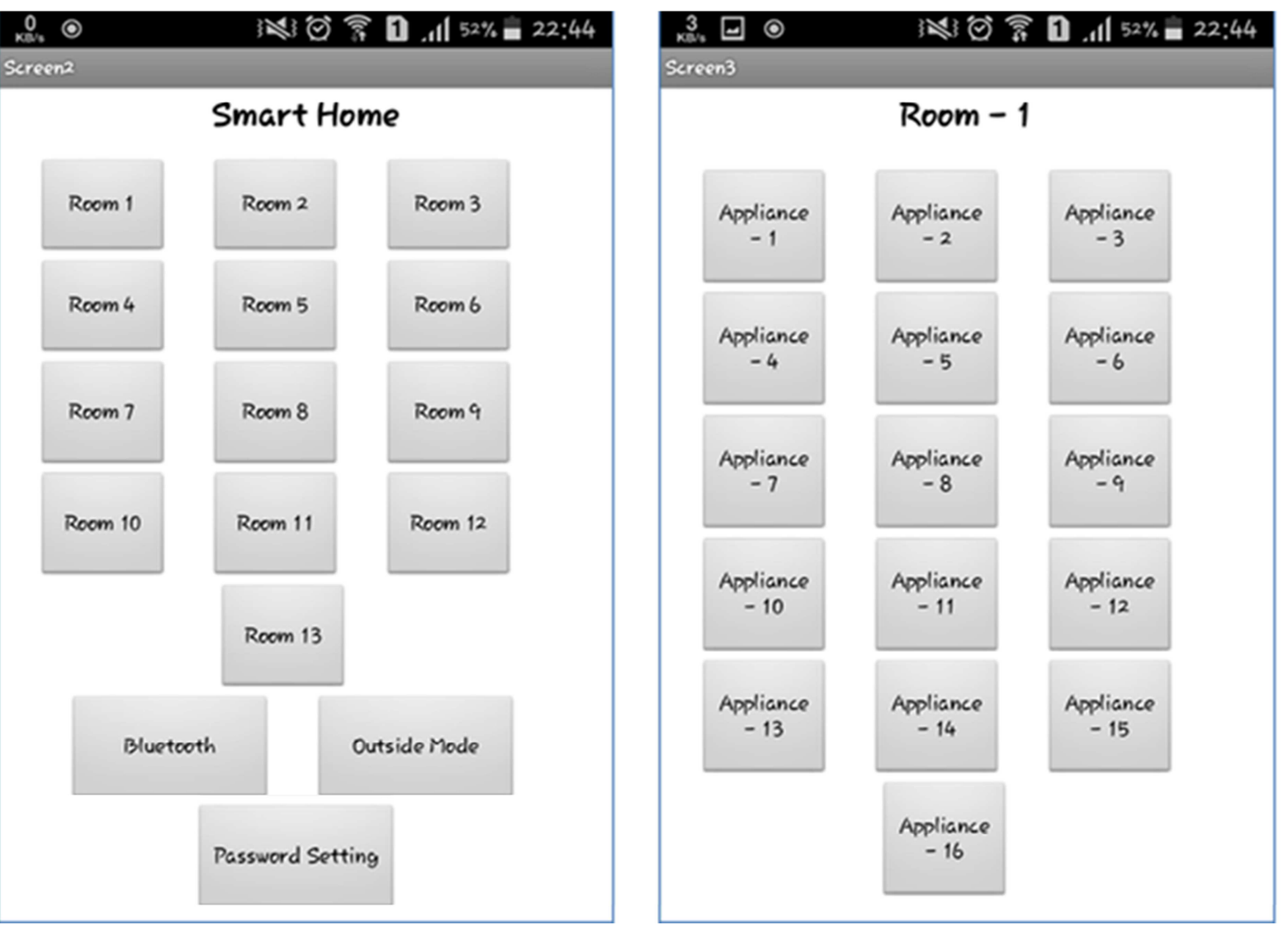

Figure 2. Smart phone application screenshot.

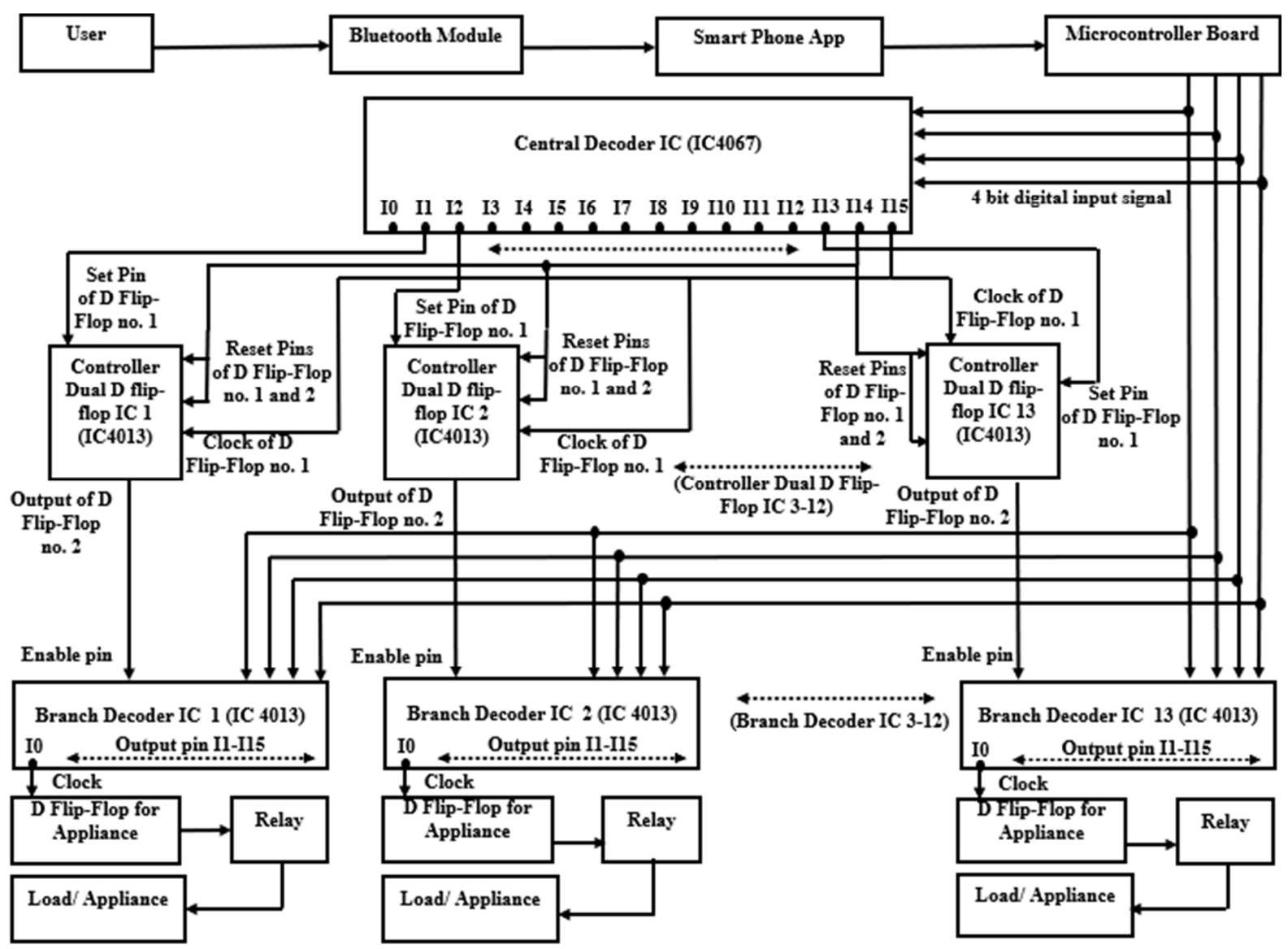

Figure 3. Block diagram of proposed appliance switching circuit. 


\section{Design Approach of Proposed Microcontroller Controlled Appliance Switching Circuit}

The proposed circuit for switching on/off appliance consists of IC4067 (4-16 line decoder IC) and IC4013 (D Flip-flop IC). The proposed circuit requires only 5 back to back 4-bit digital input signals to control 208 appliances. Microcontroller board Arduino UNO R3 is used to send the 4-bit input signal to the circuit. Arduino UNO R3 uses ATmega328 as the microcontroller. Smart phone app has been developed using MIT AppInventor by which the user can send commands. MIT AppInventor is an online and open source software by which smart phone applications can be developed without any sort of programming language [19]. Bluetooth HC-05 module has been used to receive commands from the smart phone app wirelessly.

Block diagram of the proposed external circuit is represented by figure 3 . The circuit divides the entire home into 13 different branches, locations or rooms. One decoder IC works as the central controller and the other decoder ICs work in the branches to control appliances. Each Branch Decoder IC has the capability of controlling 16 appliances. Hence, a total of 208 appliances can be controlled using the circuit.

Output pins (I1-I13) of the central decoders are connected to controller Dual D Flip-Flop ICs (IC 4013). IC4013 consists of two D Flip-Flops (D Flip-Flop no. 1 and D Flip-Flop no. 2). Outputs from controller D Flip-Flop ICs are connected to the Enable pin of Branch Decoder ICs. Therefore, the controller Dual D Flip-Flop ICs are used to enable the Branch Decoder IC for a particular location depending on command. Two output pins (I14 and I15) of the central decoder are used as input to the controller D Flip-Flop ICs for performing the operation of enabling Branch Decoder ICs.

Output pins of the Branch Decoder ICs are connected to the clock input pins of D Flip-Flops which are set to operate in Toggle Mode. For instance, if the output state of a positive edge triggered D Flip-Flop is Low or 0 , then upon receiving a high clock pulse, the output of the D Flip-Flop will be changed to High or 1. Output of D Flip-Flops is connected to relays driving electrical appliances.

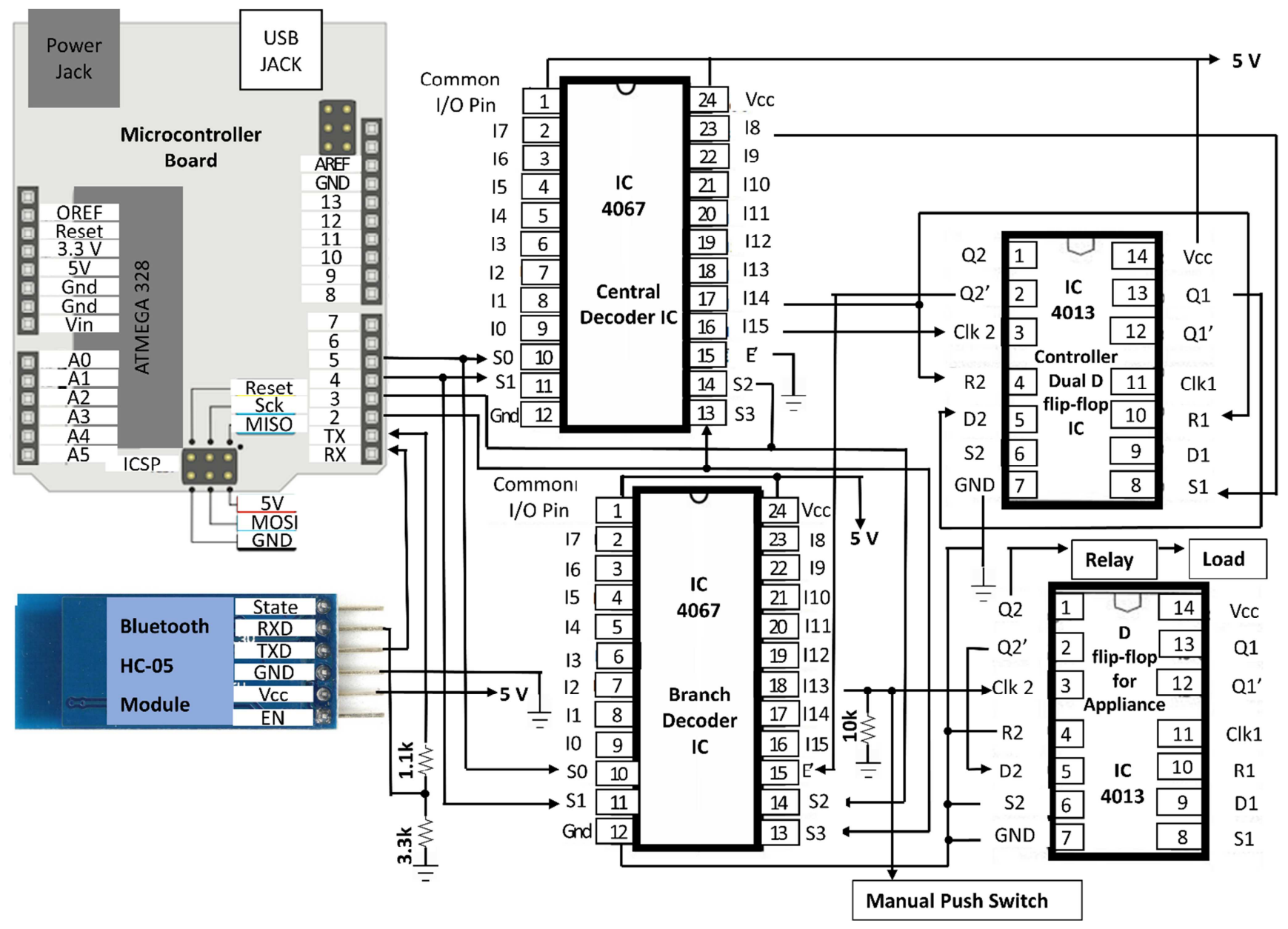

Figure 4. Schematic of proposed circuit for controlling an appliance. 


\section{Method of Operation of the Proposed Circuit}

The proposed circuit needs 5 back to back 4-bit pulse as input. Depending of the first round of 4-bit signal, one of the output pins (I1-I13) of the central decoder goes High. This makes the Set input High of D Flip-Flop no. 1 of a controller D Flip-Flop IC. This initiates the operation of the controller Dual D Flip-Flop IC. On receiving the second round of 4-bit input signal, D Flip-Flop no. 2 of that controller D Flip-Flop IC is clocked by using the I15 output pin of the Central Decoder IC. This this makes the inverted output pin of this D Flip-Flop Low and makes the corresponding Branch Decoder enabled. The third round of the 4-bit input signal makes an output pin of that Branch Decoder High which triggers the clock of D Flip-Flop connected to it. As a result, the output state of the D Flip-Flop changes which switch on/off its corresponding appliance by using relay. The fourth 4-bit input signal makes pin I14 of the central decoder High which resets both D Flip-Flops of the Controller D Flip-Flop IC. This makes the Branch IC disabled which was enabled after receiving the second round of 4-bit input signal. The final round of 4-bit signal makes the system ready to receive new command from the user.

To have a clear understanding of the operation of the proposed circuit, an example is provided using figure 4 . If the user wants to switch on/off the appliance number 13 in branch 9 , at first, he/she needs to give command from the android app by touching the particular button. The command is sent to the microcontroller board wirelessly by Bluetooth module. Since the Branch IC number is 9 for the command, the microcontroller sends the binary signal 1001 to the circuit. Initially, the branch ICs are deactivated or disabled and the central Decoder IC is activated. Therefore, the Central Decoder IC gets the signal and its pin number 23 gets High which makes the Set input of the D flip-flop (part of Controller D Flip-Flop IC) connected to it High. This result in making pin 13 of that D flip-flop go high. The second 4-bit input signal comprises of binary sequence 1111 which makes pin 16 of central decoder high. This triggers the clock (pin 3) of D flip flop which makes its pin number 2 (inverted output pin) low. This results in making the branch IC 9 enabled since its pin number 15 is getting a binary 0 . The next incoming 4 - bit input signal's bit combination is 1101 which makes the pin number 18 (output pin) of Branch IC 9 go high. This triggers the clock of the D flip-flop connected to it. This D flip-flop is operating in toggle mode and its output changes on receiving a clock pulse. The output of this D flip-flop is connected to a relay which is connected to the load. The fourth input signal bit combination is 1110 which makes pin number 17 of the central decoder High. This resets the both D Flip-Flops of the Controller D Flip-Flop IC by which the branch ICs gets disenabled or disabled. The final bit combination is 0000 and the system gets ready to receive new inputs.

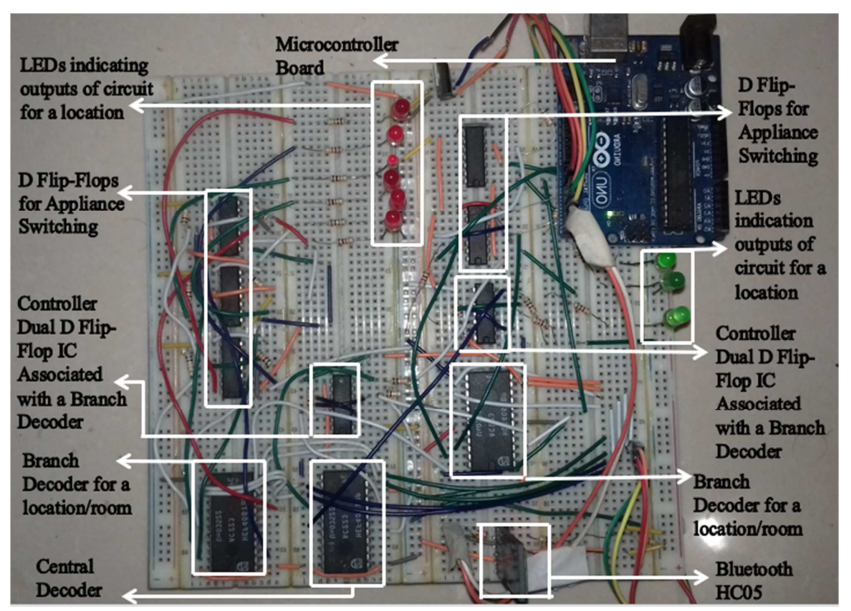

Figure 5. Practical implementation of the proposed circuit.

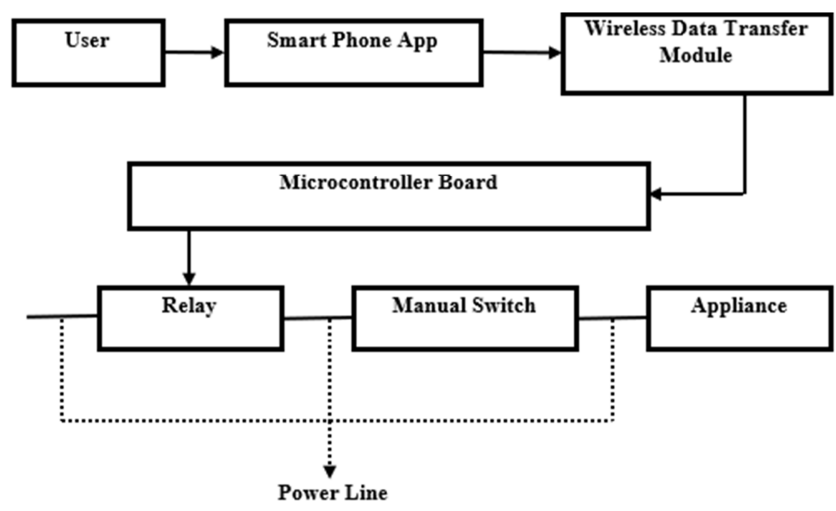

Figure 6. Problem of existing microcontroller-controlled appliance switching in case of incorporating manual switch.

\section{Design Implementation}

The proposed appliance switching circuit for microcontroller controlled smart home system has been practically implemented and tested. Picture of the implemented circuit is represented by figure 5. To show different modules clearly, the circuit contains control mechanism for only 9 appliances located in two different locations. Hence, two Branch Decoder ICs has been used. More appliances in different locations can be controlled by adding more Branch Decoder ICs and D Flip-Flop ICs. Outputs of D Flip-Flops for appliance are connected to LEDs which indicate outputs of our circuit. These output pins are required to be connected to relay for appliance switching. For controlling appliances manually, manual push switches can be connected to the clock of D Flip-Flops connected to relay.

\section{Comparison Between Proposed and Existing Designs}

The proposed appliance switching circuit can control large number of appliances compared to the conventional microcontroller systems. The circuit can incorporate manual switch which removes another major problem of existing systems. Detailed analysis between proposed and existing systems is discussed in the following sub-sections. 


\subsection{Appliance Switching Capacity}

The proposed ATmega328 microcontroller controlled have the capability of controlling 208 appliances whereas typical systems [11, 16, 19] can control only 12 appliances. ATmega2560 based system in [18] has the ability of controlling 52 appliances but still it would require 4 such kind of microcontroller to match the appliance switching capability of the proposed circuit.

\subsection{Practical Implementation in Home}

According to theoretical analysis, the microcontrollercontrolled systems [4, 5, 11, 13-19] might look very effective. However, these systems have a common major problem. These systems cannot incorporate manual switches with them. This problem is explained using figure 5. If we look fig. 6 carefully, we can observe that the microcontroller-controlled appliance switching technique won't work if the manual switch is turned off. On the contrary, if the relay switch is not energized, it is not possible to switch on the appliance using manual switch. Therefore, appliance switching has to be completely depended smart phone app, gesture, voice or sending SMS. But appliance switching cannot be completely depended on the system and adding manual switches with the system is compulsory.

The proposed circuit has the ability of adding manual switches with it. For manual control of appliances, manual push switches are needed to be connected to the clock of the D Flip-Flops for appliances. Hence, the proposed circuit overthrows a major limitation of existing microcontroller-controlled systems.

\subsection{Comparative Cost Analysis}

The proposed circuit requires only 5 wires to control 16 appliances in a location. But the existing systems need 1 wire for controlling an appliance. Hence, wiring cost is reduced by around $60-70 \%$. Moreover, hardware cost of the proposed system is also quite less than the existing microcontroller- controlled systems. A detailed cost of hardware of the proposed system and existing systems is listed in Table 1.

\subsection{System Size and Area}

After observing Table 1, the thing that might come in one's mind that the proposed circuit contains too much D Flip-Flop ICs [20]. But with careful analysis, it can be figured out that the conventional systems $[4,11,16,18]$ actually require more hardware since they will require additional hardware and circuit techniques to incorporate manual switches. However, the proposed circuit already has the hardware by which in can be incorporated with manual push switches. Moreover, the proposed circuit requires just 4 pins of a microcontroller for 208 appliances whereas the existing system [18] requires 4 microcontroller boards and existing systems in the studies [4, $11,16,19]$ require 7 microcontroller boards. Hence, size of the proposed system is actually less than the conventional microcontroller-controlled systems.

\section{Conclusion}

Microcontroller based smart home systems offer consumers a low-cost implementation of automated home. However, due to the major problems such as low appliance switching capability, problems in adding manual switches, inefficient wiring technique etc. limited widespread use of such sort of systems. The proposed circuit for appliance switching has overthrown theses major problems and have made microcontroller controlled smart home systems more reliable, more practical and cheaper. Therefore, people having little budget would be able to afford smart home systems. Since the proposed circuit can handle large number of appliances, it can be also used in industries and offices for remote switching of appliances. We believe that with the proposed appliance control circuit, road to widespread use of low-cost microcontroller controlled smart home systems will be opened.

Table 1. Hardware Cost of Proposed System and Conventional Microcontroller Controlled systems for switching on/off 208 appliances.

\begin{tabular}{llll}
\hline Proposed Appliance Switching Circuit & & & \\
\hline Product Name & Unit Price (USD) & Quantity & Sub-total (USD) \\
\hline Atmega328 based Microcontroller Board (Arduino UNO R3) & 7.5 & 1 & 7.5 \\
Bluetooth Module (HC-05) & 4.00 & 1 & 4.00 \\
D-Flip Flop IC (IC4013) & 0.18 & 117 & 21.06 \\
4-16 line Decoader IC (IC4067) & 0.94 & 14 & 13.16 \\
Total Cost=45.72 USD & & & \\
\hline
\end{tabular}

\begin{tabular}{llll}
\hline Existing Appliance Switching Systems in [4, 11, 16] & & & Sub-total \\
\hline Product Name & Unit Price (USD) & Quantity & 127.5 \\
\hline Atmega328 based Microcontroller Board (Arduino UNO R3) & 7.5 & 17 & 4.00 \\
Bluetooth Module (HC-05) & 4.00 & 1 & \\
Total Cost=131.5 USD & & & \\
\hline & & Quantity & Sub-total \\
\hline Existing Appliance Switching System in [18] & Unit Price (USD) & 4 & 5.84 \\
\hline Product Name & 14.46 & 1 & 8.00 \\
\hline Atmega2560 based Microcontroller Board (Arduino Mega 2560) & 8.00 & & \\
Wi-fi Router & & & \\
Total Cost=65.84 & &
\end{tabular}




\section{References}

[1] A. Pnevmatikakis, "Recognising Daily Functioning Activities in Smart Homes," Wireless Personal Communications, vol. 96, no. 3, pp. 3639-3654, October 2017.

[2] M. Hasan, P. Biswas, M. T. I. Bilash and M. A. Z. Dipto, "Smart Home Systems: Overview and Comparative Analysis," 2018 Fourth International Conference on Research in Computational Intelligence and Communication Networks (ICRCICN), Kolkata, India, 2018, pp. 264-268.

[3] T. M. Ladwa, S. M. Ladwa, R. S. Kaarthik, A. R. Dhara and N. Dalei, "Control of remote domestic system using DTMF," International Conference on Instrumentation, Communication, Information Technology, and Biomedical Engineering 2009, Bandung, 2009, pp. 1-6.

[4] S. Kumar, S. R. Lee, "Android based smart home system with control via bluetooth and internet connectivity," The 18th IEEE International Symposium on Consumer Electronics (ISCE), JeJu Island, 2014, pp. 1-2.

[5] F. Baig, S. Beg, M. F. Khan and S. J. Nawaz, "A Method to Control Home Appliances Based on Writing Commands Over the Air," Journal of Control, Automation and Electrical Systems, vol. 26, no. 4, pp. 421-429, 2016.

[6] W. M. Khan and I. A. Zualkernan, "SensePods: A ZigBee-Based Tangible Smart Home Interface," in IEEE Transactions on Consumer Electronics, vol. 64, no. 2, pp. 145-152, May 2018.

[7] S. Zhihua, "Design of Smart Home System Based on ZigBee," 2016 International Conference on Robots \& Intelligent System (ICRIS), Zhangjiajie, 2016, pp. 167-170.

[8] J. V. Lee, Y. D. Chuah and C. T. Chai, "A Multilevel Home Security System (MHSS)," International Journal of Smart Home, vol. 7, no. 2, pp. 49-60. 2013.

[9] M. Bassoli, V. Bianchi, I. D. Munari, P. Ciampolini, "An IoT Approach for an AAL Wi-Fi Based Monitoring System," IEEE Transactions on Instrumentation and Measurement, vol. 66, no. 12, pp. 3200-3209, 2017.

[10] M. Hasan, M. H. Anik and S. Islam, "Microcontroller Based Smart Home System with Enhanced Appliance Switching Capacity," in 2018 Fifth HCT Information Technology Trends, Dubai, United Arab Emirates, 2018, pp. 364-367.
[11] R. Piyare, M. Tazil, "Bluetooth based home automation system using cell phone," 2011 IEEE 15th International Symposium on Consumer Electronics (ISCE), Singapore, 2011, pp. 192-195.

[12] F. Y. Melhem, O. Grunder, Z. Hammoudan and N. Moubayed, "Optimization and Energy Management in Smart Home Considering Photovoltaic, Wind, and Battery Storage System With Integration of Electric Vehicles," in Canadian Journal of Electrical and Computer Engineering, vol. 40, no. 2, pp. 128-138, Spring 2017.

[13] V. Puri and A. Nayyar, "Real time smart home automation based on PIC microcontroller, Bluetooth and Android technology," 2016 3rd International Conference on Computing for Sustainable Global Development (INDIACom), New Delhi, 2016, pp. 1478-1484.

[14] G. M. S. Mahmud Rana, A. A. Mamun Khan, M. N. Hoque and A. F. Mitul, "Design and implementation of a GSM based remote home security and appliance control system," 2013 2nd International Conference on Advances in Electrical Engineering (ICAEE), Dhaka, 2013, pp. 291-295.

[15] H. AlShu'eili, G. S. Gupta and S. Mukhopadhyay, "Voice recognition based wireless home automation system," 2011 4th International Conference On Mechatronics (ICOM), Kuala Lumpur, 2011, pp. 1-6.

[16] S. Gunputh, A. P. Murdan and V. Oree, "Design and implementation of a low-cost Arduino-based smart home system," 2017 IEEE 9th International Conference on Communication Software and Networks (ICCSN), Guangzhou, 2017, pp. 1491-1495.

[17] Y. Wenbo, W. Quanyu and G. Zhenwei, "Smart home implementation based on Internet and WiFi technology," 2015 34th Chinese Control Conference (CCC), Hangzhou, 2015, pp. 9072-9077.

[18] M. Kusriyanto and B. D. Putra, "Smart home using local area network (LAN) based arduino mega 2560," 2016 2nd International Conference on Wireless and Telematics (ICWT), Yogyakarta, 2016, pp. 127-131.

[19] W. S. Yoo, S. A. Shaik, "Development of home management system using arduino and appinventor," Computer Software and Application Conference (COMPSAC), 2016 IEEE 40th annual, Atlanta, GA, June 2016, pp. 279-280.

[20] M. Hasan, M. J. Hossein, U. K. Saha and M. S. Tarif, "Overview and Comparative Performance Analysis of Various Full Adder Cells in $90 \mathrm{~nm}$ Technology," $20184^{\text {th }}$ International Conference on Computing, Communication and Automation (ICCCA), Greater Noida, India, 2018, pp. 1-6. 